\title{
Tibial Metastasis from Muscle Invasive Bladder Carcinoma: An Unusual Site
}

\author{
David Brennan Michael E. Kelly Gregory J. Nason \\ Coilin Collins-Smyth Barry B. McGuire Gerald M. Lennon \\ Department of Urology, St Vincent's University Hospital, Elm Park, Dublin, Ireland
}

\section{Key Words}

Bladder neoplasm • Carcinoma $\cdot$ Bone metastasis

\begin{abstract}
We report a case of a 61-year-old gentleman who presented with frank hematuria with associated weight loss and on-going left knee pain. Subsequent investigation revealed a muscle invasive bladder carcinoma with a related unusual bone metastasis. Though bone metastases form bladder carcinoma are common, frequent deposition sites include the spinal column and pelvis. This case report is to the best of our knowledge the first reported case of a tibial metastasis for relevant bladder carcinoma. Furthermore, we reviewed the literature, relevant diagnostic and management surrounding such occurrences.

Copyright $\odot 2013$ S. Karger AG, Basel
\end{abstract}

\section{Introduction}

Bladder cancer is a common urological cancer with significant morbidity and mortality. It is the 9th most common cancer worldwide, with approximately 130,000 deaths per year [1]. Though bone metastases have been observed with bladder cancer, they are considerably less common when compared to prostate and breast cancer. Common bone metastases sites for bladder carcinoma include pelvis and spine [2]. Our case reports an unusual metastasis to the tibia.

\section{KARGER}

Fax +4161306 1234

E-Mail karger@karger.ch

www.karger.com
(C) 2013 S. Karger AG, Basel

1015-9770/13/0073-0160\$38.00/0

Accessible online at:

www.karger.com/cur

\section{Case Report}

A 61-year-old gentleman and a current smoker with a background history of hypertension and current smoker (45 pack/year history) presented with 3-week history of painless frank hematuria with associated weight loss (10 $\mathrm{kg}$ ). Initial ultrasound revealed hydronephrosis of the left kidney with chronic cortical thinning. Creatinine at presentation was $110 \mu \mathrm{mol} / \mathrm{l}$. Cystoscopy revealed an irregular raised lesion at the left ureteric junction. He proceeded to have a transurethral resection of the bladder tumor. Histology confirmed a poorly differentiated urothelial carcinoma with prominent squamous differentiation. The tumor infiltrated muscle extensively (pT2G3). Computerised tomography (CT) scan of thorax/abdomen/pelvis demonstrated a distal ureteric mass extending to left vesico-ureteric junction with severe left hydronephrosis. No other sign of metastasis was noted.

The patient also reported a 6-week history of left knee pain, requiring regular analgesia, disrupting his sleep and restricting daily activities. Plain film radiographs of the knee showed a $3.4 \mathrm{~cm}$ subtle lytic lesion involving the medial portion the left tibia (fig. 1). Radionuclide whole body bone scan demonstrated intense uptake at this point, with no other bony infiltration. Magnetic resonance imaging (MRI) displayed a $4.1 \mathrm{~cm}$ lesion with extensive marrow edema, consistent with metastasis (fig. 2). A bone biopsy of this lesion confirmed high grade carcinoma with papillary morphology and squamous differentiation, consistent with metastatic urothelial carcinoma.

Michael E. Kelly

Department of Urology, St Vincent's University Hospital Dublin 4 (Ireland)

E-Mail kellym11@tcd.ie 


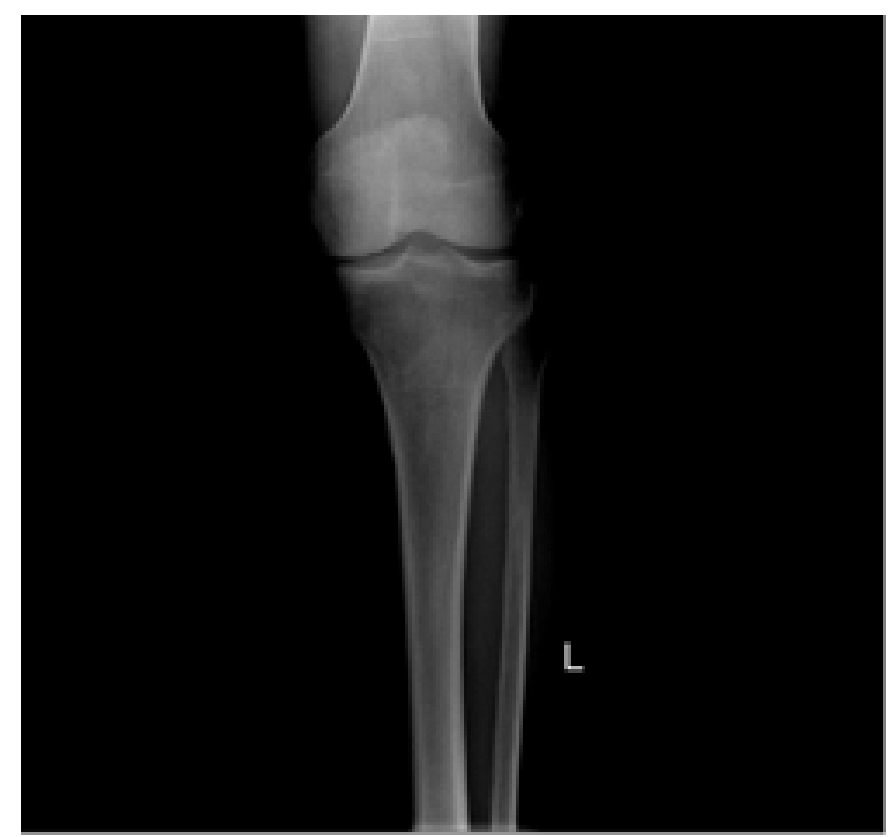

Fig. 1. Subtle lytic lesion involving the medial portion the left tibia.

Following discussion at the multi disciplinary meeting, he underwent a left total knee replacement and was commenced on a course of chemotherapy (cisplatin and gemcitabine). On review in the clinic at 2 months, he is mobile and pain free. He is undergoing regular surveillance cystoscopy.

\section{Discussion}

Transitional cell carcinoma (TCC) represents the vast majority of malignant bladder tumors. The most frequent site of metastases of TCC is to lymph nodes (51-88\%), lung (36-40\%), bone (22-37\%) and adrenal glands (6$21 \%$ ) [2]. Data suggests that bone metastasis represent a surrogate to the degree of muscle invasion of the bladder neoplasm [2]. About $30 \%$ of patients at presentation have muscle invasive disease, with $10-15 \%$ having metastatic disease evident [3]. Another $30 \%$ of newly diagnosed bladder neoplasms are likely to have occult metastatic disease [3].

The spine and pelvis are the most common bony metastatic deposit sites. Hematogenous seeding is the most common source for bladder neoplasm metastasis [4]. It

Tibial Metastasis from Bladder Carcinoma

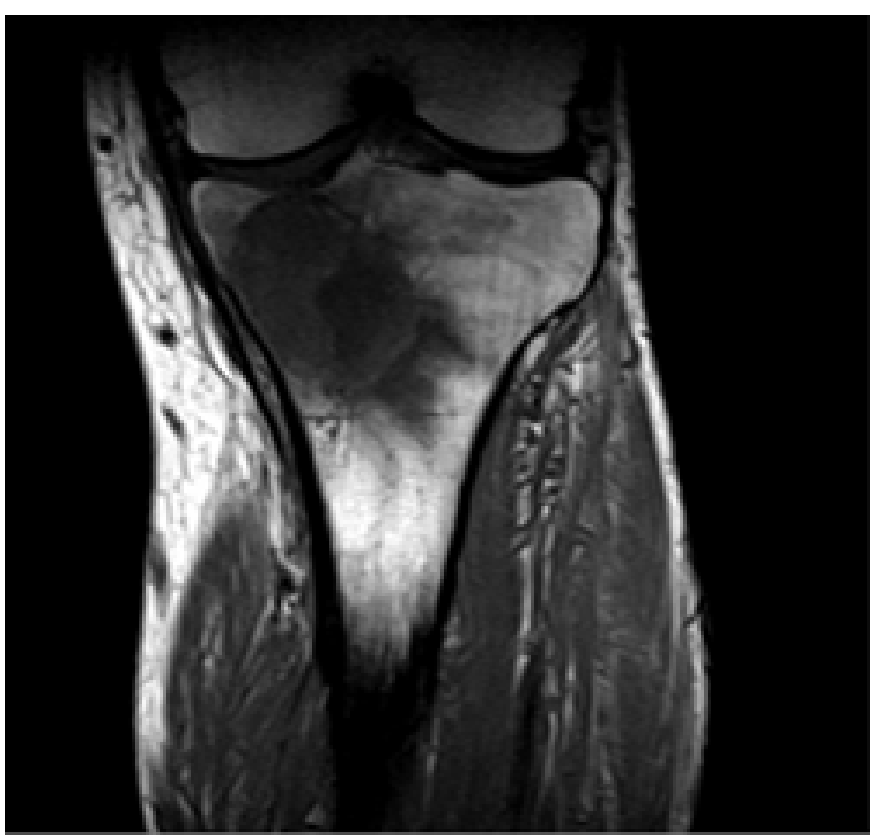

Fig. 2. MRI displaying a large lesion with extensive marrow edema, consistent with metastasis.

has been postulated that the propensity for bladder and other urological malignancies to disseminate to the spine is due to the anatomical relationship between the urological venous system and the large network of vertebral veins known as Batson's plexus [5].

Typical presentation of bone metastasis includes extensive bone pain with subsequent loss of function. Imaging is integral in assessing risk of pathological fracture. Conventional imaging has been largely replaced with higher imaging modality including CT or MRI scans. Isotope bone scan, though frequently used due to high sensitivity, lacks the specificity of CT or MRI [6]. Bone biopsy is often a viable option (under CT guidance) if there is any discrepancy in diagnosis or results changing management strategy [6].

The presence of bony involvement in bladder neoplasm indicates an advanced disease process. Median survival of between 6 and 12 months has been reported with bone metastasis [7]. Management of bony metastasis includes pain control with analgesia and/or radiotherapy. Orthopedic consultation is crucial for pre-emptive surgical management to stabilize and maintain functional status. Mirel's score highlights those that would benefit from prophylactic fixation of long bones with metastatic 
lesions. This score is based on the lesion site, size, pain severity and nature (lytic, sclerotic or mixed) [8]. Prophylactic fixation has been associated with significant symptom relief. Our case represents an unusual location of bone metastasis from TCC of the bladder, to the best of our knowledge this is the first reported case of a tibial metastasis.

\section{Conclusion}

Muscle-invasive bladder cancer carries a substantial risk of bone metastasis. This should be suspected in all patients presenting with bone pain and/or loss of function. Clinicians should be aware of atypical presentations of bone metastasis, as well as the importance of early orthopedic review in management of these patients.

\section{References}

$>1$ Ploeg M, Aben KK, Kiemeney LA: The present and future burden of urinary bladder cancer in the world. World J Urol 2009;27:289293.

2 Taher AN, Kotb MH: Bone metastases in muscle-invasive bladder cancer. Egypt Natl Canc Inst 2006;18:203-208.

$\checkmark 3$ Matthews PN, Madden M, Bidgood KA, Fisher C: The clinicopathological features of metastatic superficial papillary bladder cancer. J Urol 1984;132:904-906.
-4 Fisher MS: Lumbar spine metastasis in cervical carcinoma: a characteristic pattern. Radiology 1980;134:631-634.

$\checkmark 5$ Nathoo N, Caris EC, Wiener JA, Mendel E: History of the vertebral venous plexus and the significant contributions of Breschet and Batson. Neurosurgery 2011;69:1007-1014.

6 Oliver TB, Bhat R, Kellett CF, Adamson DJ: Diagnosis and management of bone metastases. J R Coll Physicians Edinb 2011;41:330 338
7 Dreicer R: Locally advanced and metastatic bladder cancer. Curr Treat Options Oncol 2001;2:431-436.

$>8$ Mirels H: Metastatic disease in long bones. A proposed scoring system for diagnosing impending pathologic fractures. Clin Orthop Relat Res 1989;249:256-264. 